\title{
La diversification du système éducatif péruvien
}

Vers une articulation asymétrique des offres éducatives?

The diversification of Peru's education system. Towards unevenly joined-up education provision?

La diversificación del sistema educativo peruano. ¿Hacia una articulación asimétrica de las ofertas educativas?

\section{Martín Santos}

Traducteur : Philippe Rabaté

\section{(2) OpenEdition} Journals

Édition électronique

URL : https://journals.openedition.org/ries/6069

DOI : $10.4000 /$ ries.6069

ISSN : 2261-4265

Éditeur

France Education international

Édition imprimée

Date de publication : 1 décembre 2017

Pagination : 83-90

ISBN : 978-2-85420-616-6

ISSN : $1254-4590$

Référence électronique

Martín Santos, "La diversification du système éducatif péruvien », Revue internationale d'éducation de Sèvres [En ligne], 76 | décembre 2017, mis en ligne le 01 décembre 2019, consulté le 25 juin 2021 URL : http://journals.openedition.org/ries/6069 ; DOI : https://doi.org/10.4000/ries.6069 


\title{
La diversification du système éducatif péruvien*
}

\section{Vers une articulation asymétrique des offres éducatives?}

\author{
Martín Santos \\ Pontificia Universidad Católica del Perú
}

La topographie et le fonctionnement des systèmes scolaires dans les sociétés contemporaines peuvent être compris comme le résultat d'une relation dialectique entre une logique de différenciation des parties qui les composent et une logique d'articulation multi-scalaire (locale, régionale, nationale et globale). Dans le cas péruvien, l'on constate une différenciation croissante du type d'institutions éducatives qui dispensent l'enseignement de base ainsi que le supérieur. S'agit-il d'une différenciation fragmentée qui donne lieu à des entités éducatives autocentrées et désarticulées ou s'agit-il plutôt d'une différenciation combinée avec différents niveaux d'articulation entre les parties? Par ailleurs, est-ce que cette différenciation crée des dynamiques de stratification dans le système ? Ou bien produit-elle des dynamiques qui atténuent la stratification ? Ou les deux phénomènes à la fois ? Ces thèmes seront abordés dans le présent article.

L'éducation basique au Pérou est légalement gratuite et obligatoire, et elle comporte trois niveaux (initial, primaire et secondaire) et douze années d'étude. Le ministère de l'éducation du Pérou dirige la politique éducative du pays, autrement dit, établit le programme national, paye le salaire des enseignants et contrôle le fonctionnement du système éducatif (en particulier du secteur public).

L'expansion du système éducatif public a été l'un des phénomènes les plus importants dans la constitution de la société péruvienne au $\mathrm{XX}^{\mathrm{e}}$ siècle (Guadalupe, 2009) ${ }^{1}$ et elle apparaît comme le résultat d'un double mouvement : d'un côté, la volonté de l'État central installé à Lima, la capitale, et de l'autre, la demande des communautés urbaines et rurales qui souhaitaient s'intégrer dans l'État-nation et dans la citoyenneté (Oliart, 2014). Ces populations voyaient dans l'éducation la possibilité d'un dépassement de leur situation ("progrès ») et d'une mobilité sociale. C’est ainsi que depuis le début des années 1940 environ,

* Article traduit par Philippe Rabaté.

1. De fait, selon l'historien Contreras (1996), le XXe siècle pourrait bien être considéré comme "le siècle de l'éducation ", si l'on considère l'attente et la demande d'expansion de celle-ci. 
le nombre d'inscrits a commencé à croître, et que les années soixante ont vu une accélération de cette croissance, au point que le pourcentage des personnes ayant achevé au moins le cycle primaire a dépassé la moyenne latino-américaine (Guadalupe, 2009). Cette expansion a permis à des groupes qui étaient auparavant exclus du système éducatif de commencer à l'intégrer, principalement des populations paysannes et indigènes, les pauvres des villes et les femmes (Ames, 2016).

Or, au Pérou, l'extension de la couverture éducative ne s'est pas accompagnée d'une augmentation de la dépense en éducation, bien au contraire : dans les années soixante-dix, marqué par un contexte de réduction de la croissance économique, le budget destiné à l'éducation a baissé ${ }^{2}$. Parallèlement, le nombre d'enseignants de l'éducation primaire d'État a cru de manière disproportionnée par rapport à l'augmentation du nombre d'inscrits. La conséquence a été la détérioration de leurs salaires réels (Guadalupe, 2016) et ce processus a entraîné une détérioration de la qualité de l'éducation publique. Dans ce contexte, l'éducation privée s'est transformée en un choix alternatif pour les classes moyennes et supérieures des grandes villes du Pérou. Au fil du temps, une image s'est installée selon laquelle l'éducation privée est, en général, " meilleure » que l'éducation publique (Cuenca, 2013). Puis, dans les années 1990, durant la présidence d'Alberto Fujimori, des réformes néolibérales furent mises en place dans le champ de l'économie mais aussi de l'éducation.

\section{LIBÉRALISATION DE L’ÉdUCATION, CROISSANCE DIVERSIFIÉE DU SECTEUR PRIVÉ ET DIVERSIFICATION DE L'OFFRE ÉDUCATIVE PUBLIQUE}

Durant la présidence d'Alberto Fujimori (1990-2000), un changement profond dans la conception et le fonctionnement de l'État a eu lieu sous couvert d'une politique économique néo-libérale, dans laquelle on insistait sur l'importance de l'économie de libre-échange et sur la nécessité de limiter l'intervention étatique en économie, tandis que débutait un processus de "tertiarisation » et de «technocratisation" des fonctions de l'État (Cuenca, 2013). Dans ce cadre, des réformes de libéralisation de l'éducation furent entreprises, qui s'appuyaient sur la promotion de l'investissement privé dans l'offre éducative ${ }^{3}$ (décret législatif $\mathrm{n}^{\circ} 882$ ), au vu de l'inefficacité et la qualité très basse des services éducatifs administrés par l'État. On pensait alors que l'offre privée gèrerait le service éducatif d'une manière plus efficiente et offrirait une éducation de meilleure qualité. Des investisseurs du pays, issus de champs très différents (consortiums d'entreprises, mais également investisseurs particuliers) participèrent à la création

2. C'est seulement à la fin des années 1990 que l'on a retrouvé les niveaux que l'on avait vingt ans auparavant. Actuellement, la dépense publique destinée à l'éducation représente environ $3 \%$ du PIB.

3. Ce qui permet, par exemple, d'avoir des écoles à des fins lucratives, ou en octroyant des crédits d'impôts. 
d'institutions éducatives. Ceci allait constituer le point de départ de la croissance de l'offre privée au Pérou. Précisément, sur la période 2000-2017, l'on observe une augmentation du nombre d'inscriptions dans les collèges privés (en comparaison avec la décennie précédente), aussi bien à Lima que dans d'autres villes importantes du Pérou (Balarin, 2016a). Dans cette perspective, Cuenca (2013) précise au sujet de la capitale :

En 1998, les inscriptions privées étaient de 515000 élèves et, pour l'année 2012, ce chiffre a atteint 929000 en doublant pratiquement son volume initial. Ces quelque un million d'élèves inscrits dans les écoles privées représentent $46 \%$ de l'ensemble des inscriptions scolaires à Lima.

Il est important d'indiquer que, dans le cas de Lima, l'augmentation de l'offre en écoles privées a eu lieu dans les quartiers les plus pauvres de la ville ${ }^{4}$, quartiers qui, cependant, ont connu un fort développement économique et sont considérés, pour quelques-uns d'entre eux, comme "émergents ». Or certains auteurs (par exemple Balarin, 2016a) ont souligné la faible régulation et la supervision limitée du marché privé éducatif de la part du ministère de l'éducation. Ceci expliquerait en partie l'apparition d'écoles privées à bas coût qui ne répondraient même pas aux standards minimaux de qualité (infrastructures, qualité des professeurs, entre autres aspects). Outre cette offre privée adressée à des secteurs sociaux aux moyens limités, il en existe également une autre pour les classes moyennes et supérieures. Il existe ainsi, par exemple, des collèges privés, appelés "alternatifs ", qui s'appuient sur un modèle éducatif se présentant comme différent du modèle pédagogique conventionnel et des collèges privés destinés aux élites qui offrent, entre autres, des baccalauréats internationaux. Cuenca (2013) a décrit de manière tout à fait appropriée la profonde hétérogénéité de cette offre privée :

[...] Cette recherche démontre que l'offre privée est hétérogène. Il existe à Lima des écoles privées d'élite, aussi bien confessionnelles ou laïques qu'internationales, réparties de manière inégale parmi ses 43 quartiers. Il y en a également qui fonctionnent au moyen de partenariats public-privé, d'autres qui s'organisent de manière coopérative et d'autres, enfin, qui appartiennent à des corporations intéressées par l'éducation. Ces écoles privées peuvent aussi bien occuper des terrains de 32 hectares que des surfaces de $160 \mathrm{~m}^{2}$, et certaines familles paient 3000 sols mensuels pour l'éducation de leurs enfants tandis que d'autres s'acquittent d'une mensualité de 190 sols $^{5}$.

La dynamique de cette offre éducative privée nous conduit à nous demander si elle reproduit ou exacerbe la stratification existante dans le système éducatif péruvien, en fonction de la classe sociale des élèves (Balarin, 2016a).

4. Ainsi, pour les $20 \%$ les plus pauvres, le coût de l'inscription privée a doublé entre 1998 et 2012 en passant de $19,9 \%$ à 39,3\% (Cuenca, 2013).

5. Le nouveau sol est la monnaie péruvienne actuelle, dont le taux de conversion est approximativement de 3,80 sols pour un euro $(N d T)$. 
Nous avons dit que la dépense en éducation de la part de l'État péruvien n'avait pas évolué en fonction de l'expansion qu'a connue l'ensemble du système éducatif. Ceci a conduit à une détérioration de la qualité de l'éducation publique. En ce sens, diverses évaluations nationales et internationales récentes ${ }^{6}$ montrent que les élèves péruviens n'ont pas atteint le niveau escompté. C'est dans ce contexte qu'ont été créés les "collèges d'excellence» (Colegios de Alto Rendimiento, COAR), à destination des meilleurs élèves des écoles publiques. L'État assigne à ces institutions éducatives un plus grand volume horaire et une meilleure qualité de ressources en comparaison avec les autres écoles publiques. L'introduction de ce modèle d'école publique nous conduit de nouveau à nous demander s'il reproduit ou exacerbe la stratification existante dans le système éducatif péruvien, cette fois-ci selon le «talent » des étudiants (Ames, 2016).

\section{Collèges publics SOUS GESTION PRIVÉE : LE CAS DES ÉCOLES Fe y Alegría}

Les écoles «Fe y Alegría » constituent un mouvement international d'éducation jésuite présent dans plus de dix-sept pays et principalement en Amérique latine (Lavado et al., 2014). Ces structures d'enseignement accueillent actuellement plus de 88000 élèves qui étudient dans quatre-vingts institutions éducatives. Elles s'adressent à des enfants qui ont peu de ressources dans des zones pauvres du Pérou et leur fonctionnement s'apparente à celui d'une association public-privé, dans laquelle sont administrées les ressources humaines et physiques de quelques écoles publiques du Pérou. Lavado et al. (2014) ont mesuré l'effet que pouvait avoir le fait d'y étudier sur la réussite en mathématique et en compréhension de l'écrit. Le cas des écoles Fe y Alegría montre à nouveau la profonde hétérogénéité qui existe dans l'offre d'éducation basique au Pérou et nous laisse entendre que la proposition pédagogique et curriculaire d'une école peut être plus importante que son caractère public ou privé per se. Ainsi, Balarin (2016b) signale qu'un élément distinctif de la proposition des écoles Fe y Alegría serait de tisser une relation étroite avec les familles des élèves ${ }^{7}$. Selon cette auteure, ceci pourrait nous aider à comprendre que ces écoles, qui travaillent avec des élèves en situation économique précaire, obtiennent de meilleurs résultats que d'autres écoles publiques accueillant des élèves qui proviennent pourtant

\footnotetext{
6. Tel est le cas des évaluations sur les apprentissages réalisées par le ministère de l'éducation au cours de ces dernières années, ainsi que des épreuves internationales comme le Tercer Estudio Regional de Evaluación de la Calidad de la Educación y el OECD Programme for International Student Assessment (PISA) pour les années 2000 et 2012.

7. Le même phénomène semble également se produire dans le cas d'autres réseaux d'écoles paroissiales catholiques.
} 
des mêmes milieux. La question qui reste en suspens est la suivante : à quelles conditions peut-on reproduire le modèle "Fe y Alegría », étant donné le profil singulier des parents qui inscrivent leurs enfants dans ces collèges?

\section{PAR-DELÀ LE TYPE DE GESTION : CLASSE, STRATIFICATION ET SÉGRÉGATION DANS LA RÉUSSITE ACADÉMIQUE}

La classe sociale est un élément plus déterminant dans la configuration du système éducatif péruvien que le type de gestion scolaire. Mais à quel point l'origine sociale pèse-t-elle dans la réussite académique des élèves péruviens ? Benavides et al. (2014) ont pu établir, dans une étude comparative portant sur différents pays latino-américains soumis aux tests PISA 2000 et 2009, que le Pérou est le pays dans lequel le niveau socio-économique est à l'origine de plus grande variation ( $27 \%)$ dans la réussite des élèves. Par ailleurs, ces auteurs ont découvert que les écoles péruviennes ont augmenté leur ségrégation en termes socioéconomiques. Autrement dit, elles sont devenues beaucoup plus homogènes sur le plan interne en fonction du niveau socio-économique, tandis que la variabilité (socioéconomique) entre les différentes écoles a augmenté. Les distinctions de classe sociale sont donc une composante importante des contextes éducatifs.

\section{ÉCOLE URBAINE ET ÉCOLE RURALE : UN ÉCART CRUCIAL}

Le pays que nous connaissons sous le nom de Pérou est né de la domination coloniale imposée par les conquistadors espagnols aux habitants natifs de des contrées, qui ont été classifiés comme des "Indiens ». L'indépendance de cette possession espagnole a mis fin à la période coloniale et a permis d'établir la république dans les premières décennies du XIX ${ }^{\mathrm{e}}$ siècle. À la fin de ce même siècle, après la défaite du Pérou contre le Chili en 1879, les élites intellectuelles et politiques commencèrent à définir le problème du Pérou comme «le problème posé par les populations indiennes ». Le Pérou était alors considéré comme un pays fragmenté et désarticulé géographiquement et socialement, où il fallait intégrer et incorporer les masses indigènes analphabètes (Oliart, 2014). Au début du $\mathrm{XX}^{\mathrm{e}}$ siècle, l'éducation était en effet avant tout considérée comme une manière de " transformer » les Indiens et, comme l'indique Oliart (2014), " de cette manière, l'État pourrait “éliminer" les Indiens à travers l'éducation». Le fait est que, comme nous l'avons dit auparavant, une expansion de l'éducation de base a eu lieu au Pérou tout au long du $\mathrm{XX}^{\mathrm{e}}$ siècle. Par ailleurs, dans la seconde moitié de ce même siècle, le Pérou a subi un phénomène démographique majeur : les migrations de la campagne vers la ville ont bouleversé le visage du pays, qui est passé du statut 
de rural à urbain. Actuellement, selon le recensement national de l'année 2007, le Pérou est constitué à plus de $70 \%$ de personnes vivant dans des zones urbaines. En outre, au cours des trois dernières décennies, l'on a commencé à reconnaître le caractère multiculturel et plurilingue du pays dans la Constitution et les différentes lois péruviennes. Dans ce contexte, le ministère de l'éducation du Pérou a établi l'éducation interculturelle bilingue dans les zones du pays (par exemple, des zones rurales) où l'on parle majoritairement une langue originelle (le quechua, pour n'en citer qu'une). Dans ces écoles, le programme est enseigné en langue indigène et le castillan est utilisé comme langue seconde. Toutefois, en dépit de ces changements dans la politique éducative, des écarts subsistent : les élèves des populations autochtones ( indigènes ») et rurales connaissent des taux de redoublement, d'abandon et de retard plus importants que leurs pairs dont la langue maternelle est le castillan (Ames, 2016). Ces mêmes élèves présentent un taux de réussite académique (mesuré par les tests standardisés) bien plus bas que ceux de leurs pairs de langue castillane. À cet égard, Guerrero et al. (2012) ont pu établir que les écoles créées pour accueillir des enfants parlant une langue originelle disposent de moins de services basiques et d'infrastructures que les écoles urbaines. Dans le même sens, Cueto et al. (2016) ont relevé que les enseignants de ces écoles reçoivent un appui pédagogique moins fort, disposent de connaissances pédagogiques moindres et font face à des taux d'assiduité scolaire plus bas que leurs pairs des écoles urbaines, aussi bien publiques que privées.

Pourquoi ces écarts persistent-ils ? La réponse est liée, de manière décisive, à la présence de structures sociales qui se reproduisent de manière récurrente. Comme l'a signalé fort pertinemment Ames (2016) :

Les inégalités sont présentes avant et bien après l'école : les garçons et les filles indigènes et afro-péruviens font face dans ce pays à des handicaps sociaux et économiques qui affectent indubitablement leurs apprentissages et qui mettent en évidence des structures sociales inégalitaires.

$\mathrm{Au}$ regard de ce que nous venons d'exposer, que peut faire la politique éducative nationale pour contrer ou transformer les effets de ces structures sociales dans l'expérience éducative de ces enfants qui appartiennent à des populations natives et vivent dans des zones rurales? Il s'agit de l'un des grands défis de la politique éducative au Pérou, pour lequel il n'y a pas encore de réponse politique claire qui se traduise en actions et programmes articulés.

\section{RÉFLEXIONS FINALES : DIVERSIFICATION, ARTICULATION ET DOMINATION}

Si l'on considère la situation du système éducatif péruvien que nous venons de décrire, s'agit-il d'une fragmentation de l'offre éducative qui donne lieu à des entités éducatives autocentrées et désarticulées ou s'agit-il plutôt d'une différenciation combinée avec différents niveaux d'articulation entre les parties? 
Si l'on constate indéniablement une profonde hétérogénéité, stratification et ségrégation à l'intérieur du secteur privé et du secteur public, et entre ces différents secteurs, il existe également différents niveaux d'articulation entre les parties du système : un premier niveau est lié aux grandes lignes curriculaires et légales tracées par le ministère de l'éducation du Pérou ${ }^{8}$; un second niveau renvoie à l'existence de standards éducatifs de réussite scolaire établis par les organismes internationaux comme l'OCDE et l'Unesco, qui ont été retenus comme critères par le ministre de l'éducation du Pérou et par les entités éducatives péruviennes de l'éducation de base ; un troisième niveau est lié à l'existence de réseaux d'écoles (par exemple, le réseau "alternatif », le réseau catholique, le réseau d'écoles internationales, etc.). Comme on peut le constater, il s'agit d'une articulation multi-scalaire asymétrique, qui est affectée par des relations de pouvoir et de domination (par exemple, l'hégémonie des critères de réussite scolaire établis par les organismes internationaux). Par ailleurs, cette articulation multi-scalaire asymétrique est touchée par l'intrusion de logiques d'autres systèmes sociaux : l'économie (les critères d'efficacité) et la politique (le développement d'un système national d'éducation comme objectif politique $\left.{ }^{9}\right)$.

\section{BibLIOGRAPHIE}

AMES P. (2016) : "La educación básica peruana a inicios del siglo XX : posibilidades y desafíos ", in M. Quero (coord.) : El Perú en los inicios del siglo XXI : cambios y continuidades desde las Ciencias Sociales, Universidad Nacional Autónoma de México, p. 29-44.

BALARIN M. (2016a) : «La privatización por defecto y el surgimiento de las escuelas privadas de bajo costo en el Perú. ¿Cuáles son sus consecuencias? », Revista de la Asociación de Sociología de la Educación, vol. 9, 2, p. 181-196.

BALARIN M. (2016b) : «El contexto importa : reflexiones acerca de cómo el contexto y la composición escolar afectan el rendimiento y la experiencia educativa de los estudiantes ", in Investigación para el desarrollo en el Perú. Once Balances. Lima: Grupo de Análisis para el Desarrollo, p. 27-53.

BENAVIDES M., LEON J., ETESSE M. (2014) : Desigualdades educativas y segregación en el sistema educativo peruano. Una mirada comparativa de las pruebas PISA 2000 y 2009, Lima : GRADE.

CONTRERAS C. (1996) : Maestros, mistis y campesinos en el Perú rural del siglo XX. Documentos de Trabajo, 80, Serie Historia, 16, Lima : Instituto de Estudios Peruanos.

CUENCA R. (2013) : «La escuela pública en Lima Metropolitana. ¿Una institución en extinción? ", Revista Peruana de Investigación Educativa, 5, p. 73-98.

CUETO S. et al. (2016c) : Education trajectories: from early childhood to early adulthood in Peru, Young Lives.

8. Comme nous l'avons indiqué, bien que le ministère de l'éducation du Pérou jouisse d'une capacité limitée de supervision de l'offre éducative privée, il établit toutefois le programme national et il est légalement chargé de sanctionner les entités collectives privées qui ne respecteraient pas la Loi générale de l'éducation.

9. Voir sur ce point Oliart (2014). 
GUADALUPE C. (2009): Teachers in Peruvian primary schools: views on teaching as a profession in a challenging context, these doctorale, University of Sussex, Brighton.

GUADALUPE C. (2016) : "Problemas centrales de la educación básica de cara al bicentenario nacional », in M. Quero (coord.) : El Perú en los inicios del siglo XXI: cambios y continuidades desde las Ciencias Sociales, Universidad Nacional Autónoma de México, p. 45-59.

GUERRERO G., et al. (2012) : Young Lives school survey in Peru: design and initial findings. Working Paper, 92, Oxford : Young Lives.

LAVADO P. et al. (2014): El efecto de Fe y Alegría sobre el desempeño escolar en segundo de primaria: explotando el sorteo en el ingreso como experimento natural, documento de discusión, Lima : Universidad del Pacífico y Grupo de Análisis para el Desarrollo.

OLIART P. (2014) : «Proyectos educativos y políticas », dans Modernidad y Educación en el Perú. Serie Diversidad cultural, 8, Lima : Ministerio de Cultura. 